\title{
GEOMETRIC-BASED APPROACH TO FAULT DETECTION FOR MULTILINEAR AFFINE SYSTEMS
}

\author{
F. Hamelin and T. Boukhobza
}

\author{
Research Centre in Automatic Control \\ (CRAN - CNRS UMR 7039), Henri Poincare University, \\ BP 239, 54506 Vandouvre Cedex, Nancy, France, \\ Phone: 33383684 468, Fax: 33383684 462, \\ email: frederic.hamelin@cran.uhp-nancy.fr
}

\begin{abstract}
This paper deals with the Robust Fault Detection (RFD) problem for multilinear affine uncertain systems and proposes an approach to solve it. Convex Minkowski combinations are used to compute as precisely as possible uncertainty sets according to the parameters variations in a fault-free context. Faults to be detected are characterized either by a parameter change outside its definition interval or by an additive term. The proposed approach leads to generate not only a residual function but also an optimal threshold. The detection filter associated to the residual generation is such that it minimises the effects of both parametric uncertainties and unknown input disturbances on residual while maximizing fault sensitivity. Copyright ${ }^{\complement} 2005$ IFAC
\end{abstract}

Keywords: Fault detection, residual generation, robustness, multilinear affine system.

\section{INTRODUCTION}

Fault detection problems are more and more often based on an accurate representation of the physical systems. Now, solving a RFD problem with a linear model representation implies that several kinds of model uncertainties and unknown inputs have to be considered. A fundamental part of a model-based diagnosis system is the residual generator, which needs to fulfill two requirements: have good fault sensitivity properties and be insensitive to uncertainties. During the last two decades, many approaches have been developed to improve the efficiency of residual generators (Gertler (1998); Chen and Patton (1999); Sauter and Hamelin (1999); Patton et al. (2000)). However, it is now admitted that a structured form of model uncertainty does not allow to take into account a large class of model errors, and so leads to an approximate representation of reality. In this context, this paper gives a new formulation of the RFD problem for systems depending on parametric uncertainties (Hamelin and Sauter (2000)). The latter are represented by multilin- ear affine systems with the motivation of Tan (2002). Comparatively to the ellipsoidal uncertainty, which is classically used in RFD synthesis, one may obtain considerably less conservative fault detection results using the description developed here for plants with physical parameter uncertainties. Furthermore, interval algebra has been developing several methods for the diagnosis of linear interval systems (Hamelin et al. (1999); Puig et al. (2002)). This feature allows us to detect any fault characterized by a parameter change outside its definition interval or by additive terms.

The paper is organized as follows: after section 2, which is devoted to the problem formulation, Minkowski combinations and Kharitonov polynomials are used to describe sets of uncertainty values in sections 3 and 4 . These mathematical tools help us to determine more precisely the variation domain of a residual function in a safety context. In section 5 , we detail the synthesis of a detection filter. Finally some perspectives are given in section 6 . 


\section{PROBLEM STATEMENT}

The residual generator discussed hereafter involves the classical model-based methodology. In this context, a problem of a prime importance in the design of a F.D.I. scheme is its robustness with respect to parameters uncertainties and model plant mismatches which are unavoidable in practical situations. In order to make easier the understanding of the proposed approach, a S.I.S.O. model represented by a transfer function form is considered:

$$
y(s)=G_{y u}\left(s, \theta_{u}\right) u(s)+G_{y d}\left(s, \theta_{d}\right) d(s)
$$

where $y$ represents the output, $u$ denotes the control input and $d$ represent fault-free unknown input. Transfer functions $G_{y u}\left(s, \theta_{u}\right)$ and $G_{y d}\left(s, \theta_{d}\right)$ characterize the effect distribution of the different inputs acting on the output. They depend on unknown vectors $\theta_{u}$ and $\theta_{d}$ which allow to take into account the parametric uncertainties or variations. Therefore, $\theta_{u}$ and $\theta_{d}$ represent arbitrary vectors in some bounded region of the parametric space and belong to the polytopes described by the following sets:

$$
\Theta_{u, d}=\left\{\theta_{u, d} \in \mathbb{R}^{v}, \underline{\theta_{u, d}} \leq \theta_{u, d} \leq \overline{\theta_{u, d}}\right\}
$$

A multilinear affine system corresponds to an uncertain transfer function whose numerator and denominator polynomials are multilinear affine polynomials:

$$
\begin{aligned}
G_{y u}\left(s, \theta_{u}\right) & =G_{y u}^{0}(s) G_{y u}^{1}\left(s, \theta_{u}^{1}\right) \cdots G_{y u}^{q_{u}}\left(s, \theta_{u}^{q_{u}}\right) \\
& =\frac{N_{y u}^{0}(s) N_{y u}^{1}\left(s, \theta_{u}^{1}\right) \cdots N_{y u}^{q_{u}}\left(s, \theta_{u}^{q_{u}}\right)}{D_{y u}^{0}(s) D_{y u}^{1}\left(s, \theta_{u}^{1}\right) \cdots D_{y u}^{q_{u}}\left(s, \theta_{u}^{q_{u}}\right)} \\
G_{y d}\left(s, \theta_{d}\right) & =G_{y d}^{0}(s) G_{y d}^{1}\left(s, \theta_{d}^{1}\right) \cdots G_{y d}^{q_{d}}\left(s, \theta_{d}^{q_{d}}\right) \\
& =\frac{N_{y d}^{0}(s) N_{y d}^{1}\left(s, \theta_{d}^{1}\right) \cdots N_{y d}^{q_{d}}\left(s, \theta_{d}^{q_{d}}\right)}{D_{y d}^{0}(s) D_{y d}^{1}\left(s, \theta_{d}^{1}\right) \cdots D_{y d}^{q_{d}}\left(s, \theta_{d}^{q_{d}}\right)}
\end{aligned}
$$

If $P\left(s, \theta_{u, d}^{k}\right)$ indifferently represents $N_{y u}^{k}\left(s, \theta_{u}^{k}\right)$, $D_{y u}^{k}\left(s, \theta_{u}^{k}\right), N_{y d}^{k}\left(s, \theta_{d}^{k}\right)$ or $D_{y d}^{k}\left(s, \theta_{d}^{k}\right)$, it is then assumed that coefficients $f_{i}\left(\theta_{u, d}^{k}\right)$ depend linearly on unknown vector $\theta_{u, d}^{k}$ i.e. $P\left(s, \theta_{u, d}^{k}\right)=$ $\sum_{i=0}^{n} f_{i}\left(\theta_{u, d}^{k}\right) s^{i}$.

Furthermore, all the coefficients of the multilinear affine polynomials are supposed to be independent. In this respect, $\theta_{u, d}^{k}$ must be disjoint parameters vectors. Next, the presence of a fault is characterized either by a change of vector $\theta_{u} \notin \Theta_{u}$ or by additive term $G_{y f}\left(s, \theta_{f}\right) f(s)$. The model of the system in faultycase is then represented by the following equation:

$$
\begin{gathered}
y_{f}(s)=G_{y u}\left(s, \theta_{u}^{f}\right) u(s)+G_{y d}\left(s, \theta_{d}\right) d(s) \\
+G_{y f}\left(s, \theta_{f}\right) f(s)
\end{gathered}
$$

with $\Theta_{u}^{f}=\left\{\theta_{u}^{f} \in \mathbb{R}^{v}, \underline{\theta_{u}^{f}} \leq \theta_{u}^{f} \leq \overline{\theta_{u}^{f}}\right\} \not \subset \Theta_{u}$.

According to the definition of transfer functions $G_{y u}\left(s, \theta_{u}\right)$ (3), and $G_{y d}\left(s, \theta_{d}\right)$ (4), a complete set of external residuals is generated as follows:

$$
\begin{aligned}
& e_{\operatorname{ext}}\left(j \omega, \Theta_{u}\right)= \\
& \quad D_{y u}^{0}(j \omega) D_{y u}^{1}\left(j \omega, \Theta_{u}^{1}\right) \cdots D_{y u}^{q_{u}}\left(j \omega, \Theta_{u}^{q_{u}}\right) y(j \omega)(6) \\
& -N_{y u}^{0}(j \omega) N_{y u}^{1}\left(j \omega, \Theta_{u}^{1}\right) \cdots N_{y u}^{q_{u}}\left(j \omega, \Theta_{u}^{q_{u}}\right) u(j \omega)
\end{aligned}
$$

Referring to Eq. (1) and considering parametric uncertainties, the expression describing the disturbances response in the residual set (called the internal form $e_{\text {int }}\left(j \omega, \Theta_{u, d}\right)$ of the residual generator) is given by:

$$
\begin{array}{r}
e_{\text {int }}\left(j \omega, \Theta_{u, d}\right)=D_{y u}^{0}(j \omega) D_{y u}^{1}\left(j \omega, \Theta_{u}^{1}\right) \cdots \\
D_{y u}^{q_{u}}\left(j \omega, \Theta_{u}^{q_{u}}\right) G_{y d}\left(j \omega, \Theta_{d}\right) d(j \omega)
\end{array}
$$

In the case where polytopes $\Theta_{u, d}$ are reduced to one point $\left(\theta_{u, d}=\overline{\theta_{u, d}}\right)$ then $e_{\text {ext }}\left(j \omega, \Theta_{u}\right)=$ $e_{\text {int }}\left(j \omega, \Theta_{u, d}\right)$ when no fault occurs on the system. On the other hand, if we consider uncertain parameters such that $\theta_{u, d}<\overline{\theta_{u, d}}$ then we cannot state equality $e_{\text {ext }}\left(j \omega, \overline{\left.\Theta_{u}\right)}=e_{\text {int }}\left(j \omega, \Theta_{u, d}\right)\right.$ even in fault-free case. Indeed, if no fault occurs on the system, we can only claim that $e_{\text {ext }}\left(j \omega, \Theta_{u}\right) \cap e_{\text {int }}\left(j \omega, \Theta_{u, d}\right) \neq \emptyset$.

Consequently, a simple way for detecting a fault consists in testing the intersection between sets $e_{\text {ext }}\left(j \omega, \Theta_{u}\right)$ and $e_{\text {int }}\left(j \omega, \Theta_{u, d}\right)$. If this intersection is reduced to the empty set then a fault affects the system, otherwise it is impossible to conclude about a fault occurrence because it exists particular vectors $\theta_{u, d}^{\star}$ such that: $e_{\text {ext }}\left(j \omega, \theta_{u}^{\star}\right)=e_{\text {int }}\left(j \omega, \theta_{u, d}^{\star}\right)$. In this respect, we have to evaluate or to compute sets $e_{\text {ext }}\left(j \omega, \Theta_{u}\right)$ and $e_{\text {int }}\left(j \omega, \Theta_{u, d}\right)$ as precisely as possible. Indeed, if we use too wide approximations for bounding these two sets, we will obtain an inefficient detection scheme since it won't be able to detect low amplitude faults. At this stage, it is important to notice that set $e_{\text {int }}\left(j \omega, \Theta_{u, d}\right)$ corresponds to a disc of radius $\max _{\Theta_{u, d}}\left|e_{\mathrm{int}}\left(j \omega, \Theta_{u, d}\right)\right|$ if it is assumed that $|d(j \omega)| \leq 1$. Furthermore, in practical terms, the shape of set $e_{\text {ext }}\left(j \omega, \Theta_{u}\right)$ depends on the on line estimation of spectra $u(j \omega)$ and $y(j \omega)$. It results that the study of the intersection between sets $e_{\text {ext }}\left(j \omega, \Theta_{u}\right)$ and $e_{\text {int }}\left(j \omega, \Theta_{u, d}\right)$ can be reduced to:

- the on line generation of residual $r_{1}(j \omega)$ :

$$
r_{1}(j \omega)=G_{r y}^{\star}(j \omega) \min _{\Theta_{u, d}}\left|e_{\text {ext }}\left(j \omega, \Theta_{u}\right)\right|
$$

where transfer function $G_{r y}^{\star}(s)$ is determined in order to handle fault detection while ensuring the achievement of robust performances;

- the evaluation of $r_{1}(j \omega)$ by means of threshold $T h(j \omega)$ :

$$
T h(j \omega)=\left|G_{r y}^{\star}(j \omega)\right| \max _{\Theta_{u, d}}\left|e_{\text {int }}\left(j \omega, \Theta_{u, d}\right)\right|
$$

A fault is then declared if $r_{1}(j \omega)>T h(j \omega)$, which is equivalent to $e_{\text {ext }}\left(j \omega, \Theta_{u}\right) \cap e_{\text {int }}\left(j \omega, \Theta_{u, d}\right)=\emptyset$.

The two following sections explain in details the computation of $\max _{\Theta_{u, d}}\left|e_{\text {int }}\left(j \omega, \Theta_{u, d}\right)\right|$ and $\min _{\Theta_{u, d}}\left|e_{\text {ext }}\left(j \omega, \Theta_{u}\right)\right|$. To achieve this, we use conjointly Minkowski combinations and Kharitonov polynomials.

\section{COMPUTATION OF $\underset{\Theta_{U, D}}{\operatorname{MAX}}\left|E_{\mathrm{INT}}\left(J \omega, \Theta_{U, D}\right)\right|$}

This problem can be reduced to a simple form if we refer to the Minkowski geometric algebra (Farouki 
et al. (2001)), which provides the natural extension of real interval arithmetic (Moore (1979)) to set of complex numbers.

\subsection{Minkowski combinations(Farouki et al. (2001))}

Given set operands $\mathcal{A}$ and $\mathcal{B}$, the Minkowski sum and product are the basic operations to the Minkowski geometric algebra. They are defined as follows:

$$
\begin{aligned}
& \mathcal{A} \oplus \mathcal{B}=\{a+b \mid a \in \mathcal{A} \text { and } b \in \mathcal{B}\} \\
& \mathcal{A} \otimes \mathcal{B}=\{a \times b \mid a \in \mathcal{A} \text { and } b \in \mathcal{B}\}
\end{aligned}
$$

The Minkowski operations $\oplus$ and $\otimes$ are commutative and associative but the distributive law does not hold. Furthermore, a set $\mathcal{A}$ does not have generally a multiplicative inverse. Indeed, even if Minkowski division operation is defined as $\mathcal{A} \oslash \mathcal{B}=$ $\{a \div b \mid a \in \mathcal{A}$ and $b \in \mathcal{B}\}, \oslash$ is not the inverse operation to $\otimes \operatorname{since}(\mathcal{A} \otimes \mathcal{B}) \oslash \mathcal{B} \neq \mathcal{A}$

\subsection{Kharitonov polynomials (Kharitonov (1979))}

Due to the linear dependance of $P\left(j \omega_{0}, \theta_{u, d}^{k}\right)$ upon $\theta_{u, d}^{k}$, value set $P\left(j \omega_{0}, \Theta_{u, d}^{k}\right)$ is written as:

$$
P\left(j \omega_{0}, \Theta_{u, d}^{k}\right)=\bigoplus_{i=1}^{\alpha} g_{i}\left(j \omega_{0}\right)\left(\Theta_{u, d}^{k}\right)_{i}
$$

with $g_{i}\left(j \omega_{0}\right)=a_{i}\left(j \omega_{0}\right)+j b_{i}\left(j \omega_{0}\right)$.

If $a_{i}\left(j \omega_{0}\right) b_{i}\left(j \omega_{0}\right)=0$ for all $i \in[0, \alpha]$, then the contour of the set $P\left(j \omega_{0}, \Theta_{u, d}^{k}\right)$ consists of a rectangle. The coordinates of the corners are calculated by means of the four Kharitonov polynomials:

$\left\{\begin{array}{l}K_{u, d}^{1}\left(j \omega_{0}\right)=\overline{\beta_{0}}+j \overline{\beta_{1}} \omega_{0}-\underline{\beta_{2}} \omega_{0}^{2}-j \underline{\beta_{3}} \omega_{0}^{3}+\ldots \\ K_{u, d}^{2}\left(j \omega_{0}\right)=\underline{\beta_{0}}+j \underline{\beta_{1}} \omega_{0}-\overline{\beta_{2}} \omega_{0}^{2}-j \overline{\beta_{3}} \omega_{0}^{3}+\ldots \\ K_{u, d}^{3}\left(j \omega_{0}\right)=\underline{\beta_{0}}+j \overline{\beta_{1}} \omega_{0}-\overline{\beta_{2}} \omega_{0}^{2}-j \underline{\beta_{3}} \omega_{0}^{3}+\ldots \\ K_{u, d}^{4}\left(j \omega_{0}\right)=\overline{\beta_{0}}+j \underline{\beta_{1}} \omega_{0}-\underline{\beta_{2}} \omega_{0}^{2}-j \overline{\beta_{3}} \omega_{0}^{3}+\ldots\end{array}\right.$

with $\underline{\beta_{i}}=\min _{\theta_{u, d}^{k} \in \Theta_{u, d}^{k}} f_{i}\left(\theta_{u, d}^{k}\right)$ and $\overline{\beta_{i}}=\max _{\theta_{u, d}^{k} \in \Theta_{u, d}^{k}} f_{i}\left(\theta_{u, d}^{k}\right)$.

\subsection{Computation of $P\left(j \omega_{0}, \Theta_{u, d}^{k}\right)$ in the general case}

According to definition (12), let us now assume that $a_{i}\left(j \omega_{0}\right) b_{i}\left(j \omega_{0}\right) \neq 0$ for some $i \in[0, \alpha]$. In this case, it is not sufficient to consider the four Kharitonov polynomials for computing the contour of set $P\left(j \omega_{0}, \Theta_{u, d}^{k}\right)$. It is necessary to make use of the following lemma to determine exactly the convex octagon vertices $P\left(j \omega_{0}, \Theta_{u, d}^{k}\right)$.

Lemma 1. Let us consider affine polynomial

$$
\begin{aligned}
P\left(j \omega_{0}, \theta_{u, d}^{k}\right) & =\sum_{i=0}^{n} f_{i}\left(\theta_{u, d}^{k}\right)\left(j \omega_{0}\right)^{i}=\sum_{i=1}^{\alpha} g_{i}\left(j \omega_{0}\right)\left(\theta_{u, d}^{k}\right)_{i} \\
& =\sum_{i=1}^{\alpha}\left(a_{i}\left(j \omega_{0}\right)+j b_{i}\left(j \omega_{0}\right)\right)\left(\theta_{u, d}^{k}\right)_{i}
\end{aligned}
$$

with $\theta_{u, d}^{k} \leq \theta_{u, d}^{k} \leq \overline{\theta_{u, d}^{k}}$. In the complex plane, $P\left(j \omega_{0}, \Theta_{u, d}^{k}\right)$ describes the interior of a convex polygon composed of a finite number of edges. The coordinates of the polygon's vertices can be determined as follows:

1. For all $i \in[0, \alpha]$, let us define:

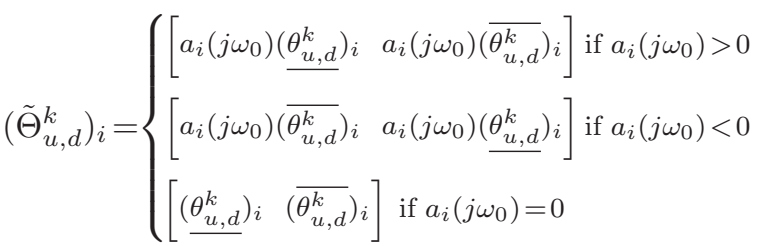

2. $P\left(j \omega_{0}, \Theta_{u, d}^{k}\right)$ is rewritten in accordance with notation $\tilde{\Theta}_{u, d}^{k}$ :

$$
\begin{aligned}
P\left(j \omega_{0}, \tilde{\Theta}_{u, d}^{k}\right)= & \overbrace{\bigoplus_{i=1}^{\beta}\left(1+j \frac{b_{i}\left(j \omega_{0}\right)}{a_{i}\left(j \omega_{0}\right)}\right)}^{\bigoplus_{i=\beta+1}^{\alpha} j b_{i}\left(j \omega_{0}\right)}\left(\tilde{\Theta}_{u, d}^{k}\right)_{i}^{k})_{i}
\end{aligned}
$$

3. After factorization by $\left(1+j \frac{b_{i}\left(j \omega_{0}\right)}{a_{i}\left(j \omega_{0}\right)}\right)$, $P\left(j \omega_{0}, \tilde{\Theta}_{u, d}^{k}\right)$ is finally given by:

$$
\begin{aligned}
& P\left(j \omega_{0}, \tilde{\Theta}_{u, d}^{k}\right)=\bigoplus_{i=1}^{\gamma}\left(1+j \frac{b_{i}\left(j \omega_{0}\right)}{a_{i}\left(j \omega_{0}\right)}\right) h_{i}\left(\tilde{\Theta}_{u, d}^{k}\right) \\
& \oplus j h_{\gamma+1}\left(\tilde{\Theta}_{u, d}^{k}\right) \\
& \text { with } h_{\gamma+1}\left(\tilde{\Theta}_{u, d}^{k}\right)=\bigoplus_{i=\beta+1}^{\alpha} b_{i}\left(j \omega_{0}\right)\left(\tilde{\Theta}_{u, d}^{k}\right)_{i} \text {. }
\end{aligned}
$$

4. Convex polygon $P\left(j \omega_{0}, \Theta_{u, d}^{k}\right)$ is composed of $2 \gamma$ edges if $\alpha=\beta$; in the contrary case, it has $2(\gamma+1)$ edges. Later on, indices $i \in[1, \gamma]$ are ordered in ascending order of $\frac{b_{i}\left(j \omega_{0}\right)}{a_{i}\left(j \omega_{0}\right)}$. The cartesian coordinates $\tilde{K}_{u, d}^{i}\left(j \omega_{0}\right)$ of vertices where the sides meet are given by solving the following recursive equation for $i \in[1, \gamma]$ :

$$
\begin{aligned}
c_{i}=\Delta h_{i}\left(\tilde{\Theta}_{u, d}^{k}\right) \times & \operatorname{sign}\left(a_{i}\left(j \omega_{0}\right)\right) \times g_{i}\left(j \omega_{0}\right) \\
& +c_{i-1}
\end{aligned}
$$

with $\Delta h_{i}\left(\tilde{\Theta}_{u, d}^{k}\right)=\max h_{i}\left(\tilde{\Theta}_{u, d}^{k}\right)-\min h_{i}\left(\tilde{\Theta}_{u, d}^{k}\right)$ and $c_{0}=0$. If the convex polygon is composed of $2(\gamma+1)$ vertices then the recursion ended with $c_{\gamma+1}=j \Delta h_{\gamma+1}\left(\tilde{\Theta}_{u, d}^{k}\right)+c_{\gamma}$.

5. The coordinates of the first $\gamma$ (resp. $\gamma+1$ for $2(\gamma+1)$ vertices $)$ points $\tilde{K}_{u, d}^{i}\left(j \omega_{0}\right)$ arranged in a anticlockwise order are given by:

$$
\tilde{K}_{u, d}^{k, i}\left(j \omega_{0}\right)=c_{i}-\frac{c_{\delta}}{2}+P\left(j \omega_{0}, \theta_{u, d}^{k \star}\right)
$$

with $\theta_{u, d}^{k \star}=0.5\left(\underline{\theta_{u, d}^{k}}+\overline{\theta_{u, d}^{k}}\right)$ and $\delta=\gamma$ (resp. $\gamma+1)$.

The coordinates of the last $\gamma$ (resp. $\gamma+1$ ) points $\tilde{K}_{u, d}^{k, i}\left(j \omega_{0}\right)$ arranged in a anticlockwise order are given by:

$\tilde{K}_{u, d}^{k, i}\left(j \omega_{0}\right)=\tilde{K}_{u, d}^{k, i-1}\left(j \omega_{0}\right)+c_{i-\delta-1}-c_{i-\delta}$ 
Elements of proof:

We won't present the proof of lemma 1 as it is based on simple Euclidean geometry techniques and is not of great interest for the sequel of the paper.

It follows from results in lemma 1 that only the right side of relationship (7) cannot be evaluated due to uncertainty in $d(s)$. However, it is possible to evaluate the maximal effect of $d(j \omega)$ on $e_{\text {int }}(j \omega)$.

\subsection{Disturbance effects on $e_{\mathrm{int}}\left(j \omega_{0}\right)$}

Since it is assumed that $\left|d\left(j \omega_{0}\right)\right| \leq 1$ for all $\omega_{0}$ then, according to definition (7), $e_{\text {int }}\left(j \omega_{0}\right)$ is in value set :

$$
\frac{\bigotimes_{k=0}^{q_{u}} D_{y u}^{k}\left(j \omega_{0}, \Theta_{u}^{k}\right) \bigotimes_{k=0}^{q_{d}} N_{y d}^{k}\left(j \omega_{0}, \Theta_{d}^{k}\right)}{\bigotimes_{k=0}^{q_{d}} D_{y d}^{k}\left(j \omega_{0}, \Theta_{d}^{k}\right)} \otimes \mathcal{D}(0,1)
$$

with $\mathcal{D}(0,1)$ the closed unit disc and

$$
\left\{\begin{array} { l } 
{ \Theta _ { u } ^ { 0 } = [ \begin{array} { l l } 
{ \theta _ { u } ^ { 0 } } & { \theta _ { u } ^ { 0 } }
\end{array} ] } \\
{ \Theta _ { d } ^ { 0 } = [ \begin{array} { l l } 
{ \theta _ { d } ^ { 0 } } & { \theta _ { d } ^ { 0 } }
\end{array} ] }
\end{array} \Rightarrow \left\{\begin{array}{l}
D_{y u}^{0}\left(j \omega_{0}, \Theta_{u}^{0}\right)=D_{y u}^{0}\left(j \omega_{0}\right) \\
N_{y d}^{0}\left(j \omega_{0}, \Theta_{d}^{0}\right)=N_{y d}^{0}\left(j \omega_{0}\right) . \\
D_{y d}^{0}\left(j \omega_{0}, \Theta_{d}^{0}\right)=D_{y d}^{0}\left(j \omega_{0}\right)
\end{array}\right.\right.
$$

Thus, $\left|e_{\text {int }}\left(j \omega_{0}\right)\right|$ is bounded in fault-free case by:

$$
\frac{\max _{\theta_{u, d}^{k} \in \Theta_{u, d}^{k}} \prod_{k=0}^{q_{u}}\left|D_{y u}^{k}\left(j \omega_{0}, \theta_{u}^{k}\right)\right| \prod_{k=0}^{q_{d}}\left|N_{y d}^{k}\left(j \omega_{0}, \theta_{d}^{k}\right)\right|}{\min _{\theta_{d}^{k} \in \Theta_{d}^{k}} \prod_{k=0}^{q_{d}}\left|D_{y d}^{k}\left(j \omega_{0}, \theta_{d}^{k}\right)\right|}
$$

From Lemma 1, $\left|e_{\text {int }}\left(j \omega_{0}\right)\right|$ can be majored by:

$$
\frac{\prod_{k=0}^{q_{u}} \max _{i}\left|\tilde{K}_{D_{y u}}^{k, i}\left(j \omega_{0}\right)\right| \prod_{k=0}^{q_{d}} \max _{i}\left|\tilde{K}_{N_{y d}}^{k, i}\left(j \omega_{0}\right)\right|}{\min _{\theta_{d}^{k} \in \Theta_{d}^{k}} \prod_{k=0}^{q_{d}}\left|D_{y d}^{k}\left(j \omega_{0}, \theta_{d}^{k}\right)\right|}
$$

To evaluate this expression, it is necessary to determine the minimal distance from polygon $D_{y d}^{k}\left(j \omega_{0}, \Theta_{d}^{k}\right)$ to the origin in the complex plane. According to the denominator of (18), it is useful to differentiate between $\min _{\theta_{d}^{k} \in \Theta_{d}^{k}}\left|D_{y d}^{k}\left(j \omega_{0}, \theta_{d}^{k}\right)\right|=0$ and $\min _{\theta_{d}^{k} \in \Theta_{d}^{k}}\left|D_{y d}^{k}\left(j \omega_{0}, \theta_{d}^{k}\right)\right| \neq 0$. This point in polygon (PIP) problem is to determine whether the origin lies inside, outside, or on the boundary of a polygon. Lemma 2 is helpful in solving this problem. But before presenting concrete details of this lemma, let us introduce integer $i_{0}, 1 \leq i_{0} \leq 2 \delta$ such that:

$$
\begin{aligned}
\arg & \left(D_{y d}^{k}\left(j \omega_{0}, \theta_{d}^{k \star}\right)-\tilde{K}_{D_{y d}}^{k, i_{0}}\left(j \omega_{0}\right)\right) \\
& \geq \arg \left(D_{y d}^{k}\left(j \omega_{0}, \theta_{d}^{k \star}\right)\right) \\
& >\arg \left(D_{y d}^{k}\left(j \omega_{0}, \theta_{d}^{k \star}\right)-\tilde{K}_{D_{y d}, i_{0}+1}^{k}\left(j \omega_{0}\right)\right)
\end{aligned}
$$

with $\theta_{d}^{k \star}=0.5\left(\underline{\theta_{d}^{k}}+\overline{\theta_{d}^{k}}\right), \tilde{K}_{D_{y d}}^{k, 2 \delta+1}\left(j \omega_{0}\right)=\tilde{K}_{D_{y d}}^{k, 1}\left(j \omega_{0}\right)$ and the coordinates of the $2 \delta$ vertices of $D_{y d}^{k}\left(j \omega_{0}, \Theta_{d}^{k}\right)$ given by $\tilde{K}_{D_{y d}}^{k, i}\left(j \omega_{0}\right)=v_{i}+j w_{i}$.

Lemma 2. $\min _{\theta_{d}^{k} \in \Theta_{d}^{k}}\left|D_{y d}^{k}\left(j \omega_{0}, \theta_{d}^{k}\right)\right| \neq 0$ iff area $S_{D_{y d}^{k}}$ of convex polygon $D_{y d}^{k}\left(j \omega_{0}, \Theta_{d}^{k}\right)$ is smaller than area $S_{\mathcal{P}}$ of polygon $\mathcal{P}$ defined by the set of points $\left\{\tilde{K}_{D_{y d}}^{k, 1}\left(j \omega_{0}\right), \ldots, \tilde{K}_{D_{y d}}^{k, i_{0}}\left(j \omega_{0}\right), 0, \tilde{K}_{D_{y d}}^{k, i_{0}+1}\left(j \omega_{0}\right), \ldots\right.$, $\left.\tilde{K}_{D_{y d}}^{k, 2 \delta+1}\left(j \omega_{0}\right)\right\}$. This property implies that: $v_{i_{0}} w_{i_{0}+1}<w_{i_{0}} v_{i_{0}+1}$

Proof of lemma 2. The lemma can be proved using the geometric structure of convex polygone $D_{y d}^{k}\left(j \omega_{0}, \Theta_{d}^{k}\right)$. On the one hand, if the origin lies

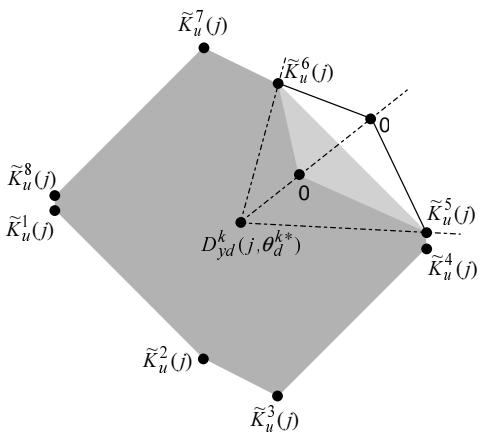

Fig. 1. Distance between $O$ and $D_{y d}^{k}\left(j \omega_{0}, \theta_{d}^{k}\right)$.

outside polygon $D_{y d}^{k}\left(j \omega_{0}, \Theta_{d}^{k}\right)$ then, according to figure $1, \mathcal{P}$ is another polygon and is such that $\mathcal{P} \supset D_{y d}^{k}\left(j \omega_{0}, \Theta_{d}^{k}\right) \Rightarrow S_{\mathcal{P}}>S_{D_{y d}^{k}}$. On the other hand, if the origin lies inside polygon $D_{y d}^{k}\left(j \omega_{0}, \Theta_{d}^{k}\right)$ then $\mathcal{P}$ has any shape with angles higher than $180^{\circ}$ and is so a concave polygon. In this case, $\mathcal{P}$ is a proper subset of $D_{y d}^{k}\left(j \omega_{0}, \Theta_{d}^{k}\right)$, that implies $S_{\mathcal{P}}<S_{D_{y d}^{k}}$. Finally, if the origin is on the boundary of $D_{y d}^{k}\left(j \omega_{0}, \Theta_{d}^{k}\right) \quad$ then $\quad \mathcal{P}=D_{y d}^{k}\left(j \omega_{0}, \Theta_{d}^{k}\right) \quad \Rightarrow$ $S_{\mathcal{P}}=S_{D^{k}}$. From these properties, $\min _{\theta_{d}^{k} \in \Theta_{d}^{k}}\left|D_{y d}^{k}\left(j \omega_{0}, \theta_{d}^{k}\right)\right| \neq 0$ iff $S_{\mathcal{P}}>S_{D_{y d}^{k}}$. The problem of determining the area of a polygon can be solved by summing the areas of a series of triangles generated by connected all the edges of the polygon to a single point. It can be shown using Green's Theorem that $2 S_{D_{y d}^{k}}=\sum_{i=1}^{2 \delta}\left(v_{i} w_{i+1}-v_{i+1} w_{i}\right)$ and $2 S_{\mathcal{P}}=\sum_{i=1, i \neq i_{0}}^{2 \delta}\left(v_{i} w_{i+1}-v_{i+1} w_{i}\right)$. Thus, $S_{\mathcal{P}}>S_{D_{y d}^{k}} \Rightarrow v_{i_{0}} w_{i_{0}+1}<w_{i_{0}} v_{i_{0}+1}$.

At this stage, if there is no $\omega_{0}$ such that $\min _{\theta_{d}^{k} \in \Theta_{d}^{k}}\left|D_{y d}^{k}\left(j \omega_{0}, \theta_{d}^{k}\right)\right| \neq 0 \forall k \in\left[0, q_{d}\right]$ then $\left|e_{\text {int }}\left(j \omega_{0}\right)\right|$ cannot be bounded. Indeed, there always exists a particular value of $\theta_{d}^{k}$ in $\Theta_{d}^{k}$ such that $\left|e_{\text {int }}\left(j \omega_{0}\right)\right|$ is infinite. In this case, it is impossible to synthesize a fault detection method avoiding false alarms. That is why, let us assume that there exists band of frequencies on which $\left|e_{\text {int }}\left(j \omega_{0}\right)\right|$ is bounded for all $\theta_{d}^{k}$ in $\Theta_{d}^{k}$. The minimal value of denominator 
(18) can be computed using lemma 3. From convexity property, the closest point to the origin of polygon $D_{y d}^{k}\left(j \omega_{0}, \Theta_{d}^{k}\right)$ is unique. To determine it, we define index $i_{1}$ and vertex $\tilde{K}_{D_{y d}}^{k, 2 \delta+2}\left(j \omega_{0}\right)$ as:

$$
\left\{\begin{array}{c}
i_{1}=\arg \min _{i \in[2,2 \delta+1]}\left|\tilde{K}_{D_{y d}}^{k, i}\left(j \omega_{0}\right)\right| \\
\tilde{K}_{D_{y d}, 2 \delta+2}^{k, 2}\left(j \omega_{0}\right)=\tilde{K}_{D_{y d}}^{k, 2}\left(j \omega_{0}\right)
\end{array}\right.
$$

Lemma 3. According to definitions (20), minimal distance $\min _{\theta_{d}^{k} \in \Theta_{d}^{k}}\left|D_{y d}^{k}\left(j \omega_{0}, \theta_{d}^{k}\right)\right|$ between origin $O$ and polygon $D_{y d}^{k}\left(j \omega_{0}, \Theta_{d}^{k}\right)$ equals:

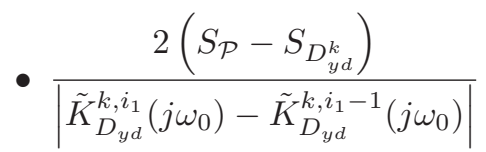

$$
\begin{aligned}
& \text { if }\left(\tilde{K}_{D_{y d}}^{k, i_{1}-1}\left(j \widehat{\omega}_{0}\right), \tilde{K}_{D_{y d}}^{k, i_{1}}\left(j \omega_{0}\right), 0\right)<\frac{\pi}{2}, \\
& \text { - } \frac{2\left(S_{\mathcal{P}}-S_{D_{y d}^{k}}\right)}{\left|\tilde{K}_{D_{y d}}^{k, i_{1}+1}\left(j \omega_{0}\right)-\tilde{K}_{D_{y d}}^{k, i_{1}}\left(j \omega_{0}\right)\right|}
\end{aligned}
$$

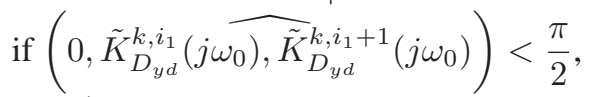

$$
\begin{aligned}
& \text { - }\left|\tilde{K}_{D_{y d}}^{k, i_{1}}\left(j \omega_{0}\right)\right| \text { otherwise. }
\end{aligned}
$$

Proof of lemma 3. The proof of this lemma is straightforward.

According to (18), maximal value of $\left|e_{\text {int }}\left(j \omega_{0}\right)\right|$ can be estimated by means of the results of lemma 3 when $\min _{\theta_{d}^{k} \in \Theta_{d}^{k}}\left|D_{y d}^{k}\left(j \omega_{0}, \theta_{d}^{k}\right)\right| \neq 0$ is bounded (lemma 2). Let us now focus on the determination of $e_{\text {ext }}\left(j \omega, \Theta_{u}\right)$.

\section{DETERMINATION OF $\underset{\Theta_{U, D}}{\operatorname{MIN}}\left|E_{\mathrm{EXT}}\left(J \omega, \Theta_{U}\right)\right|$}

To compute $\min _{\Theta_{u, d}}\left|e_{\text {ext }}\left(j \omega, \Theta_{u}\right)\right|$, it is possible either to determine the closest point to origin $O$ on polygon $e_{\text {ext }}\left(j \omega, \Theta_{u}\right)$ or to calculate the minimal distance between $N_{y u}^{0}(j \omega) N_{y u}^{1}\left(j \omega, \Theta_{u}^{1}\right) \cdots N_{y u}^{q_{u}}\left(j \omega, \Theta_{u}^{q_{u}}\right) u(j \omega)$ and $D_{y u}^{0}(j \omega) D_{y u}^{1}\left(j \omega, \Theta_{u}^{1}\right) \cdots D_{y u}^{q_{u}}\left(j \omega, \Theta_{u}^{q_{u}}\right) y(j \omega)$. The second approach is preferred because it takes considerably less calculation time. However, even if $P\left(j \omega_{0}, \Theta_{u}^{k}\right)$ is easy to determine using the results of section 3, the two-dimensional geometric shape resulting from the multiplication of polygons is complex.

To make easy the determination of this shape, circular complex arithmetic can be used. Indeed, a simple way to compute the Minkowski product $P\left(j \omega_{0}, \Theta_{u}^{k}\right) \otimes \ldots$ $\otimes P\left(j \omega_{0}, \Theta_{u}^{k-1}\right)$ consists in approximating each polygon $P\left(j \omega_{0}, \Theta_{u}^{k}\right)$ by circumcircle $\mathcal{C}_{u}^{k}\left(\mathcal{Z}_{u}^{k}, \mathcal{R}_{u}^{k}\right)$. The latter by definition is the smallest circle which contains the polygon completely within it. Center $\mathcal{Z}_{u}^{k}$ of this circumcircle is known as the polygon's circumcenter. It corresponds to the centroid (the center of mass or center of gravity) of the polygon. Circumradius $\mathcal{R}_{u}^{k}$ of circumcircle $\mathcal{C}_{u}^{k}\left(\mathcal{Z}_{u}^{k}, \mathcal{R}_{u}^{k}\right)$ is equal to the polygon radius and is defined as the maximum half distance between any two points of the polygon.

According to these definitions and (16), coordinates of centre $\mathcal{Z}_{u}^{k}$ and radius $\mathcal{R}_{u}^{k}$ are given by:

$$
\left\{\begin{array}{c}
\mathcal{Z}_{u}^{k}=\frac{\tilde{K}_{u}^{k, i_{2}}\left(j \omega_{0}\right)+\tilde{K}_{u}^{k, i_{3}}\left(j \omega_{0}\right)}{2} \tilde{K}_{u}^{k, i_{3}}\left(j \omega_{0}\right) \mid \\
\mathcal{R}_{u}^{k}=\frac{\mid \tilde{K}_{u}^{k, i_{2}}\left(j \omega_{0}\right)-\tilde{K}^{2}}{2}
\end{array}\right.
$$

with $\left(i_{2}, i_{3}\right)=\arg \max _{i<j}\left|\tilde{K}_{u}^{k, i}\left(j \omega_{0}\right)-\tilde{K}_{u}^{k, j}\left(j \omega_{0}\right)\right|$.

When all the polygons $P\left(j \omega_{0}, \Theta_{u}^{k}\right)$ are approximated by circumcircles $\mathcal{C}_{u}^{k}\left(\mathcal{Z}_{u}^{k}, \mathcal{R}_{u}^{k}\right)$, it can be shown that the Minkowski product of two circles is the area between the two loops of a Cartesian oval (Farouki and Pottmann (2002)). Instead of computing the exact Minkowski product of circles $\mathcal{C}_{u}^{k}\left(\mathcal{Z}_{u}^{k}, \mathcal{R}_{u}^{k}\right) \mathcal{C}_{u}^{k+1}\left(\mathcal{Z}_{u}^{k+1}, \mathcal{R}_{u}^{k+1}\right)$, it is useful to use another approach based on the definition of a bounding disk with center $\tilde{\mathcal{Z}}_{u}^{k}$ and radius $\tilde{\mathcal{R}}_{u}^{k}$ such that:

$$
\left\{\begin{array}{l}
\tilde{\mathcal{Z}}_{u}^{k}=\mathcal{Z}_{u}^{k} \mathcal{Z}_{u}^{k+1} \\
\tilde{\mathcal{R}}_{u}^{k}=\left|\mathcal{Z}_{u}^{k}\right| \mathcal{R}_{u}^{k+1}+\left|\mathcal{Z}_{u}^{k+1}\right| \mathcal{R}_{u}^{k}+\mathcal{R}_{u}^{k} \mathcal{R}_{u}^{k+1}
\end{array}\right.
$$

By extension, let $\quad \tilde{\mathcal{C}}_{N_{y u}}\left(\tilde{\mathcal{Z}}_{N_{y u}}, \tilde{\mathcal{R}}_{N_{y u}}\right)$ and $\tilde{\mathcal{C}}_{D_{y u}}\left(\tilde{\mathcal{Z}}_{D_{y u}}, \tilde{\mathcal{R}}_{D_{y u}}\right)$ be the bounding disks which correspond to the products $N_{y u}^{0}\left(j \omega_{0}\right) \mathcal{C}_{N_{y u}}^{1}\left(\mathcal{Z}_{N_{y u}}^{1}, \mathcal{R}_{N_{y u}}^{1}\right) \cdots \mathcal{C}_{N_{y u}}^{q_{u}}\left(\mathcal{Z}_{N_{y u}}^{q_{u}}, \mathcal{R}_{N_{y u}}^{q_{u}}\right)$ and $D_{y u}^{0}\left(j \omega_{0}\right) \mathcal{C}_{D_{y u}}^{1}\left(\mathcal{Z}_{D_{y u}}^{1}, \mathcal{R}_{D_{y u}}^{1}\right) \cdots \mathcal{C}_{D_{y u}}^{q_{u}}\left(\mathcal{Z}_{D_{y u}}^{q_{u}}, \mathcal{R}_{D_{y u}}^{q_{u}}\right)$ respectively. With these notations, the minimal distance between $O$ and $e_{\text {ext }}\left(j \omega_{0}, \Theta_{u}\right)$ with respect to $\Theta_{u}$ can be estimated by:

$\min _{\Theta_{u, d}}\left|e_{\text {ext }}\left(j \omega_{0}, \Theta_{u}\right)\right|=\left|\mathcal{Z}_{N_{y u}} u\left(j \omega_{0}\right)-\mathcal{Z}_{D_{y u}} y\left(j \omega_{0}\right)\right|$
$-\mathcal{R}_{N_{y u}}\left|u\left(j \omega_{0}\right)\right|-\mathcal{R}_{D_{y u}}\left|y\left(j \omega_{0}\right)\right|$

The previous development shows us that the complexity of the fault detection method depends on the kind of representation used to account polygons $N_{y u}^{k}\left(j \omega_{0}, \Theta_{u}^{k}\right)$ and $D_{y u}^{k}\left(j \omega_{0}, \Theta_{u}^{k}\right)$. In the particular case where the interval plant family $G_{y u}\left(s, \Theta_{u}\right)$ can be represented by a linear affine system of the form:

$$
G_{y u}\left(s, \Theta_{u}\right)=\frac{N_{y u}^{0}(s) N_{y u}^{1}\left(s, \Theta_{u}^{1}\right)}{D_{y u}^{0}(s) D_{y u}^{1}\left(s, \Theta_{u}^{1}\right)}
$$

the external residual $e_{\text {ext }}\left(j \omega_{0}, \Theta_{u}\right)(6)$ is given by:

$$
\begin{gathered}
e_{\operatorname{ext}}\left(j \omega_{0}, \Theta_{u}\right)=D_{y u}^{0}\left(j \omega_{0}\right) D_{y u}^{1}\left(j \omega_{0}, \Theta_{u}^{1}\right) y\left(j \omega_{0}\right) \\
-N_{y u}^{0}\left(j \omega_{0}\right) N_{y u}^{1}\left(j \omega_{0}, \Theta_{u}^{1}\right) u\left(j \omega_{0}\right)
\end{gathered}
$$

According to this simple definition, it appears that it is not necessary to consider circumcircles $\mathcal{C}_{N_{y u}}^{1}\left(\mathcal{Z}_{N_{y u}}^{1}, \mathcal{R}_{N_{y u}}^{1}\right)$ and $\mathcal{C}_{D_{y u}}^{1}\left(\mathcal{Z}_{D_{y u}}^{1}, \mathcal{R}_{D_{y u}}^{1}\right)$ to represent convex polygons $N_{y u}^{1}\left(j \omega_{0}, \Theta_{u}^{1}\right)$ and $D_{y u}^{1}\left(j \omega_{0}, \Theta_{u}^{1}\right)$. Indeed, even if the calculation of the minimum distance between two polygons is rather complex, it can be recast as a configuration space problem (Cameron and Culley (1986)) or it can be determined in studying anti-podal pairs between the polygons. According to these geometric methods, the minimal translational distance between two convex 
polygons can be computed and the determination of $\min _{\Theta_{u, d}}\left|e_{\text {ext }}\left(j \omega_{0}, \Theta_{u}\right)\right|$ only needs an estimation of the spectra $y\left(j \omega_{0}\right)$ and $u\left(j \omega_{0}\right)$ in realtime.

\section{RESIDUAL GENERATOR}

Let us consider the system modelled by Eq. (5). In order to minimize the energy of residual $r_{1}(j \omega)$ in fault free case and to maximize its sensitivity to additive faults, $G_{r y}^{\star}(s)$ is synthesized according to performance index $J_{1}\left(G_{r y}, \Theta_{d, f}\right)$ :

$$
\begin{array}{r}
J_{1}\left(G_{r y}, \Theta_{d, f}\right)=\frac{\int_{0}^{\infty}\left|\hat{G}_{r y}(j \omega) G_{y d}\left(j \omega, \Theta_{d}\right)\right| d \omega}{\int_{0}^{\infty}\left|\hat{G}_{r y}(j \omega) G_{y f}\left(j \omega, \Theta_{f}\right)\right| d \omega} \\
=\frac{\int_{0}^{\infty}\left|\hat{G}_{r y}(j \omega)\right|\left|e_{\text {int }}\left(j \omega, \Theta_{u, d}\right)\right| d \omega}{\int_{0}^{\infty}\left|\hat{G}_{r y}(j \omega)\right|\left|e_{\text {fault }}\left(j \omega, \Theta_{u, f}\right)\right| d \omega}
\end{array}
$$

with $e_{\text {fault }}\left(j \omega, \Theta_{u, f}\right)=$

$D_{y u}^{0}(j \omega) D_{y u}^{1}\left(j \omega, \Theta_{u}^{1}\right) \cdots D_{y u}^{q_{u}}\left(j \omega, \Theta_{u}^{q_{u}}\right) G_{y f}\left(j \omega, \Theta_{f}\right)$. Detection filter $G_{r y}^{\star}(s)$ corresponds to the solution of the following optimisation problem:

$$
G_{r y}^{\star}(j \omega)=\min _{G_{r y}} \max _{\Theta_{d, f}} J_{1}\left(G_{r y}, \Theta_{d, f}\right)
$$

It can be computed using lemma 4 which generalizes the solution given by Sauter and Hamelin (1999) with parametric uncertainties.

Lemma 4. Optimal detection filter $G_{r y}^{\star}(j \omega)$ solution of problem (25) is defined by the relation:

$$
G_{r y}^{\star}(j \omega)=\delta\left(j \omega_{0}^{*}\right)
$$

where $\delta\left(j \omega_{0}^{*}\right)$ is such that for any transfer function $H(s)$ (with $\left.H\left(j \omega_{0}^{*}\right) \neq 0\right)$, we have:

$\int_{0}^{\infty}\left|\delta\left(j \omega_{0}^{*}\right)\right|^{2} d \omega=1$ and $\left\{\begin{array}{l}\left|\delta\left(j \omega_{0}^{*}\right) H\left(j \omega_{0}^{*}\right)\right|^{2} \neq 0 \\ \left|\delta\left(j \omega_{0}^{*}\right) H(j \omega)\right|^{2}=0, \forall \omega \neq \omega_{0}^{*}\end{array}\right.$

and $\omega_{0}^{*}$ minimises the cost function:

$$
\frac{\max _{\Theta_{d}}\left|G_{y d}\left(j \omega, \Theta_{d}\right)\right|}{\min _{\Theta_{f}}\left|G_{y f}\left(j \omega, \Theta_{f}\right)\right|}
$$

\section{CONCLUSION}

In this paper, the RFD problem is analyzed for multilinear affine systems. Parametric uncertainties are considered and represented by a polytopic rectangular description. It is assumed that all the parameters of the system belong to an interval. The presented approach allows us to detect either a change in the values of these parameters outside their definition intervals or additive faults. The synthesis is based on an accurate description of the uncertain transfer functions in the complex plane. The Minkowski geometric algebra is referred to provide the natural extension of real interval arithmetic to set of complex numbers. According to these value sets, a residual is generated in order to maximize fault sensitivity and robustness with respect to parameters uncertainties. Further developments concern the application of this methodology to real process in order to detect and isolate variations in physical parameters.

\section{REFERENCES}

Cameron, S.A. and R.K. Culley (1986). Determining the minimum translational distance between two convex polyhedra, 591-596, Proc. of IEEE International Conference on Robotics and Automation.

Chen, J. and R.J. Patton (1999). Robust model-based fault diagnosis for dynamic systems, volume 3 of Kluwer international series on Asian studies in computer and information science. Kluwer Academic Publishers, Boston.

Farouki, R.T., H.P. Moon and B. Ravani (2001). 'Minkowski Geometric Algebra of Complex Sets', Geometriae Dedicata, 85, 283-315.

Farouki, R.T. and H. Pottmann (2002). 'Exact Minkowski products of $\mathrm{N}$ complex disks', Reliable Computing, 8, 43-66.

Gertler, J.J. (1998). Fault detection and diagnosis in engineering systems, Marcel Dekker, New York.

Hamelin, F., C. Defranoux and F. Rambeaux (1999). A geometric approach for fault detection and isolation in dynamic uncertain systems, 3122-3127, Proc. of IEEE Conference on Decision and Control, Phœnix, Arizona (USA).

Hamelin, F. and D. Sauter (2000). 'Robust fault detection in uncertain dynamic systems', Automatica, 36(11), 1747-1754.

Kharitonov, V.L. (1979). 'Asymptotic stability of an equilibrium position of a family of systems of linear differential equations', Translation in Differential Equations, 44, 1483-1485.

Moore, R.E., 'Methods and applications of interval analysis', volume 2 of SIAM studies in applied mathematics. Society for Industrial and Applied Mathematics SIAM, Philadelphie, 1979.

Patton, R.J., P.M. Frank and R. Clark, Issues of fault diagnosis for dynamic systems. Springer-Verlag, London, New York, 2000.

Puig, V., J. Quevedo, T. Escobet and S. de las Heras (2002). Passive robust fault detection approaches using interval models, Proc. of World Congress IFAC, Barcelona, Spain.

Sauter, D. and F. Hamelin (1999). 'Frequency-domain optimisation for robust fault detection and isolation in dynamic systems', IEEE Transactions on Automatic Control, 44(4), 878-882.

Tan, N. (2002). 'Computation of the frequency response of multilinear affine systems', IEEE Transactions on Automatic Control, 47(10), 1691-1696. 